\title{
Practical Applications of Sperm Selection Techniques as a Tool for Improving Reproductive Efficiency
}

\author{
J. M. Morrell ${ }^{1}$ and H. Rodriguez-Martinez ${ }^{2}$ \\ ${ }^{1}$ Division of Reproduction, Department of Clinical Sciences, Swedish University of Agricultural Sciences-SLU, Box 7054, \\ 75007 Uppsala, Sweden \\ ${ }^{2}$ Department of Clinical and Experimental Medicine, Faculty of Health Sciences, Linköping University, 58185 Linköping, Sweden
}

Correspondence should be addressed to J. M. Morrell, jane.morrell@kv.slu.se

Received 30 April 2010; Accepted 23 June 2010

Academic Editor: Kyle Orwig

Copyright ( 2011 J. M. Morrell and H. Rodriguez-Martinez. This is an open access article distributed under the Creative Commons Attribution License, which permits unrestricted use, distribution, and reproduction in any medium, provided the original work is properly cited.

\begin{abstract}
Modern biotechnologies are used extensively in the animal breeding industry today. Therefore, it is essential that sperm handling procedures do not modulate the normal physiological mechanisms occurring in the female reproductive tract. In this paper, the different selection mechanisms occurring in vivo are described briefly, together with their relevance to artificial insemination, followed by a detailed description of the different selection processes used in reproductive biotechnologies. These selection methods included fractionated semen collection, cryopreservation, biomimetic sperm selection, selection based on hyaluronic acid binding, and last, but not least, sperm sex selection. Biomimetic sperm selection for AI or for cryopreservation could improve pregnancy rates and help to reverse the decline in fertility seen in several domestic species over the recent decades. Similarly, selection for hyaluronic acid binding sites may enable the most mature spermatozoa to be selected for IVF or ICSI.
\end{abstract}

\section{Introduction}

Modern biotechnologies such as artificial insemination (AI) and sperm cryopreservation have revolutionized the animal breeding industry. However, to unleash the full potential of these undoubtedly powerful technologies, the events that take place in the female reproductive tract between AI and fertilization should be considered, so that we do not inadvertently hinder, or even counteract, normal physiological processes $[1,2]$. Moreover, in recent years there may have been a decline in fertility in some species, associated with an increase in the use of AI, for example in dairy cattle [3]. The aim of this paper is to examine the ways in which spermatozoa are "selected" during preparation for AI and how these processes may impact sperm fertility. First, the different selection mechanisms in the female reproductive tract will be examined briefly, followed by a discussion of the relevance of these mechanisms to AI. Sperm selection with regard to cryopreservation will be considered followed by a critique of different methods for sperm selection, with particular focus on Single Layer Centrifugation (SLC). The effectiveness of selecting spermatozoa for in vitro fertilization (IVF) and intracytoplasmic sperm injection (ICSI) according to possession of hyaluronic acid binding sites will be discussed. Lastly, sperm selection for assisted reproduction (ART) according to content of sex chromosomes will be evaluated.

\section{Selection Mechanisms within the Female Reproductive Tract}

At ejaculation, billions of spermatozoa are released into the female reproductive tract and start to make their way to the site of fertilization. However, only a few thousand spermatozoa are present in the oviducts at any one time, and only one spermatozoon will eventually fertilize each oocyte. As spermatozoa pass along the female reproductive tract they are subjected to several selection mechanisms responsible for filtering out immature or damaged spermatozoa [1], at the same time as they are acquiring fertilizing ability. Thus, only spermatozoa that are functionally normal should reach the 
potential site of fertilization in the oviducts, the isthmicampullary junction.

(i) The cervix: semen deposition takes place in the vagina, close to the posterior cervix, in human beings and some other species, for example, ruminants and rodents. In other species, for example, horses and dogs, where semen deposition takes place directly into the uterus, the uterotubal junction acts as the only filter. In species with vaginal deposition of semen, the cervix acts as a sperm reservoir where the spermatozoa may reside for up to $24 \mathrm{~h}$, and it also serves as a filtration system. Immotile or morphologically abnormal spermatozoa become trapped by the viscous mucous in the cervical lumen [4] whereas normal motile spermatozoa are thought to orientate along the long axis of mucosal threads, aligning themselves along the longitudinal mucosal folds of the cervix (where the mucus is basically composed of sialic acid) for antegrade movement [5]. Damaged spermatozoa and microorganisms are trapped in the highly viscous central mucous which propels them in a retrograde direction for subsequent expulsion. During the stages of the oestrus cycle under progesterone dominance, cervical mucous is viscous and nearly impenetrable to spermatozoa.

(ii) The uterus: although sperm-uterine interaction has been studied mostly in the pig, it is believed that many of the observations in this species are pertinent to other species too. It has been shown that some spermatozoa attach to the uterine epithelium whereas others remain in the uterine lumen, the latter tending to have damaged plasma membranes [6]. Moreover, using uterine explants, spermatozoa attached to uterine epithelial cells (UEC) were shown to have normal ultrastructure and mitochondrial membrane potential $[7,8]$. Thus, it has been suggested that the uterus, like the cervix, may act as a sperm reservoir and filtration mechanism, although the latter is based on direct interactions between the spermatozoa and the uterine epithelial cells (UECs) rather than on a physical barrier.

(iii) The uterotubal junction (UTJ) and distal isthmus may constitute a third sperm reservoir and filtering system. When biopsies were taken from this region at various times after mating, Mburu et al. [9] identified bound and nonbound sperm populations. In a later study the bound spermatozoa were found to have better membrane viability than the unbound group [10]. Further work with explants revealed that bound spermatozoa have both a prolonged lifespan [11] and better chromatin integrity [12] compared to those that do not bind. It is thought that spermatozoa that have temporarily bound to UEC or the epithelial cells of the UTJ subsequently become free to pass along the oviducts in an antegrade direction.

(iv) oviductal selection: oviductal proteins modify the zona pellucida (ZP) of freshly ovulated oocytes, thus affecting the ability of spermatozoa to interact with them. Incubation of human spermatozoa in the presence of oviductal proteins apparently reduces their affinity for the ZP [13], enabling the oviduct to actively select sperm subpopulations with specific ligands for fertilization.

(v) Selection for a functional acrosome and binding sites. sperm penetration of the cumulus matrix surrounding the oocyte in the cumulus oophorus complex is facilitated by sperm membrane-bound hyaluronidase [14] and by their hyperactivated motility pattern. Zona binding involves a wide range of sperm surface components and at least one of the three glycoproteins of which the ZP is composed. Zona binding acts as a trigger for the acrosome reaction (AR). The spermatozoon then enters the perivitelline space between the $\mathrm{ZP}$ and the oolemma, and the tip of the sperm makes contact with the oolemma, followed by lateral attachment of the spermatozoa [14]. The site of fusion is at the central region of the spermatozoa near to, or at, the equatorial region, and involves an integrin on the oocyte and an integrin ligand on the sperm plasma membrane.

\section{Relevance of Physiological Selection to Artificial Insemination}

In $\mathrm{AI}$, semen doses containing lower sperm numbers than are normally present in the ejaculate are deposited in the uterus or cervix during oestrus [1]. This site of semen deposition is nonphysiological for ruminants, bypassing the cervical sperm reservoir in these species. This means, in effect, that the person preparing the semen doses is acting as a partial substitute for the cervix, diluting the seminal plasma and selecting only those ejaculates with low proportions of immotile or morphologically abnormal spermatozoa to be used in AI. However, the presence of some seminal plasma in the uterus is nonphysiological in ruminants and may have a negative effect on fertility. Furthermore, the spermatozoa remain in contact with seminal plasma (SP) for much longer in the extended semen than they would under physiological conditions. SP contains decapacitating factors to prevent the spermatozoa capacitating too soon relative to the potential time of fertilization. Prolonged contact with SP may, therefore, impact negatively on sperm functionality. However, the type of semen extender used may also affect the ability of the spermatozoa to interact with the female reproductive tract [7].

The prolonged contact between stallion spermatozoa and seminal plasma during cooled storage may partially account for the lower pregnancy rates achieved in mares inseminated on the day following semen collection compared with AI using freshly ejaculated semen. Although semen extenders are used to dilute the seminal plasma, the formulations currently in use for stallion semen may not be sufficiently effective in maintaining the spermatozoa in an optimal condition for fertilization.

Conversely, SP also contains components that are considered beneficial to sperm survival [15]. Proteins in SP have various effects promoting or inhibiting sperm functions including motility, oviduct binding, zona binding/penetration, and ultimately fertilization [16]. Spermadhesins, for example, bind to the sperm surface on ejaculation and prevent capacitation [16]. It is speculated that removal of these proteins during preparation techniques such as flow cytometric sperm sexing, may destabilize the membrane and precapacitate the spermatozoa, thus shortening their functional life $[15,17]$. 
Once the spermatozoa have been deposited in the uterus, they are subjected to the previously described selection mechanisms and interactions with the female reproductive tract. Therefore, it is essential that the sperm handling techniques prior to sperm deposition do not compromise sperm membranes, sperm motility or sperm longevity, since this will impair their ability to progress up the female reproductive tract.

\section{Sperm Selection for Freezing}

Some individuals produce ejaculates that are better able to withstand cryopreservation than others, that is, with higher cryosurvival. In the semen industry, it is usual to select animals that are "good" freezers as breeding sires, since the market for straws of frozen semen with poor viability is nonexistent. However, this selection of sires inevitably impacts on the gene pool, since only spermatozoa surviving cryopreservation contribute their DNA to the next generation. Thus, by actively selecting for cryogenic spermatozoa, there may have been inadvertent selection for undesirable traits, such as reduced fertility.

Cryopreservation can have a profound effect on sperm viability and function, both because of the physical changes occurring during ice crystal formation and dissolution, and due to the effects of the cryopreservation medium on sperm membrane composition. Furthermore, cryopreservation may severely reduce or block binding proteins on the sperm surface that are necessary for binding to oviductal epithelium [1]. Frozen-thawed spermatozoa exhibit "capacitation-like" membrane changes, which may be evidence of membrane damage as well as actual capacitation. Reactive oxygen species, such as the superoxide anion $\left(\mathrm{O}_{2}{ }^{-}\right)$, are produced during capacitation in all species, and possibly also $\mathrm{ONOO}^{-}$in bovines [18], accompanied by increased activity of the mitochondrial respiratory chain. The additional sperm energy requirements needed to support these activities must be supplied by oxidative substrates in the surroundings, namely, the semen extender in stored semen doses. Thus the composition of the cryopreservation medium is important for supporting capacitation.

Whether cryosurvival ("freezability") is a function of the spermatozoa themselves or of the composition of the seminal plasma is the subject of much discussion. The boar ejaculate is produced in several fractions, each containing a different proportion of spermatozoa and SP, the latter being of different composition in the various fractions depending on the relative contributions of the accessory glands [19]. Spermatozoa present in the first $10 \mathrm{~mL}$ of the sperm-rich fraction of boar ejaculates (known as portion 1 or P1) were found to survive cryopreservation better than spermatozoa in the rest of the ejaculate (portion 2, P2) [20]. Moreover, when spermatozoa in the different fractions were separated from SP and subsequently mixed with SP from the other fraction in a crossover experiment, the kinematics of P2 spermatozoa were improved whereas those of P1 spermatozoa deteriorated [21]. In contrast, boar spermatozoa collected from the whole of the sperm-rich fraction were found to have better motility than those in $\mathrm{P} 1, \mathrm{P} 2$, or P1 subsequently mixed with $\mathrm{P} 2(\mathrm{P} 1+\mathrm{P} 2)[22]$. Moreover, the sperm-rich fractions of stallion ejaculate were found to contain spermatozoa with lower \%DFI (DNA fragmentation index) than those from the subsequent (spermpoor) fractions [23]. Taken together, these observations suggest that spermatozoa are affected by the composition of $\mathrm{SP}$, and at least boar and stallion spermatozoa may require exposure to SP in the correct ratio (or relative amounts of specific constituents) to achieve their optimum motility and membrane integrity.

This finding could have implications for enhancing sperm survival in species such as the stallion, where there are marked differences between individuals in sperm survival during cryopreservation or even during cooled storage. Removal of some [24], or all, of the SP [25, 26], prolongs stallion sperm survival during cooled storage. In contrast to possible negative effects of SP on sperm survival, SP may play a role in the postmating clearance of spermatozoa from the uterus, at least in mares [27]. Ejaculation in most other species is not differentiated into clear fractions, as it is in the boar and stallion, but even so there may be individual variation in the composition of SP that modulate cryosurvival. Identification of the components of SP that enhance fertility, either by their action on spermatozoa or on the female reproductive tract, would be of considerable benefit to the animal AI industry.

An alternative method for enhancing sperm survival during storage, either cooled or by cryopreservation, would be to select subpopulations of good quality spermatozoa prior to storage. Preliminary results suggest that sperm fertility is not adversely affected by such selection methods and may be improved, for example stallion spermatozoa [28]. The selection of the best quality spermatozoa is reviewed in the next section. However, it is pertinent to note here that stallion spermatozoa selected by SLC through Androcoll-E on the basis of sperm quality show increased progressive motility after thawing than unselected spermatozoa, which (it is speculated) could result in better pregnancy rates after AI with frozen thawed sperm doses.

\section{Biomimetic Sperm Selection}

Biomimetics is the use of technologies and/or processes that mimic a naturally occurring event. Several mechanisms have been suggested that mimic the selection taking place in the female reproductive tract [29]. These methods either separate the spermatozoa from SP or, in addition, permit selection of the better quality spermatozoa from the rest of the ejaculate. There have been several extensive reviews recently on the merits and demerits of various sperm selection techniques used to improve sperm quality for assisted reproduction, for example, for human spermatozoa [30], and for animal spermatozoa [29].

Basically, the methods can be divided into those that result in removal of seminal plasma only (sperm washing), and methods that select spermatozoa on the basis of certain characteristics, such as sperm migration (based on sperm 
motility), filtration (based on membrane integrity), and colloid centrifugation (based on sperm motility, morphology, viability and chromatin integrity). Colloid centrifugation can be subdivided into density gradient centrifugation (DGC) and Single Layer Centrifugation (SLC) [31]. A brief description of each of these techniques follows.

5.1. Sperm washing. The extended ejaculate is centrifuged, the supernatant (consisting of seminal plasma and extender) removed and the sperm pellet resuspended in a suitable volume of the extender. The spermatozoa are thus separated from most of the seminal plasma [32]. However, there is no selection of the spermatozoa and dead, moribund, and abnormal cells [33] as well as sources of reactive oxygen species (ROS), may remain, which are detrimental to sperm viability. There have been reports of chromatin damage in human spermatozoa due to this technique [34], although this may be due to centrifuging human semen without extender rather the technique itself. Extenders for animal semen, particularly milk- or egg yolk-based extenders, typically contain antioxidants which may counteract ROS released during centrifugation.

5.2. Migration. There are several variations, all relying on the ability of motile spermatozoa to move from one suspension to another [34]. The original sperm population is either underneath, on top of, or to one side of the migration medium [34]. The spermatozoa move away from the SP; thus selection is based on sperm motility and does not provide any selection based on morphology, chromatin integrity, viability, or acrosome integrity [35]. Spermatozoa with tail abnormalities are prevented from migrating into the swim-up medium. Some studies show significantly better midpieceand tail-morphology after swim-up than after washing [33]. Adding hyaluronic acid to the migration medium may also select for spermatozoa with intact membranes [36]. The main disadvantage of any migration method is the lowrecovery rate, for example, $10 \%-20 \%$ [33], which renders them impractical for preparing spermatozoa for $\mathrm{AI}$ in most animal species.

5.3. Filtration. The spermatozoa interact with the filter substance, for example, glass fibres, Sephadex beads, or membrane pores, but are also selected on the basis of being ability to move [37]. Nonviable spermatozoa adhere to the matrix more than motile, functional spermatozoa [38], although the mechanism of action is uncertain [39]. Immotile and dead spermatozoa may agglomerate because of changes in surface charges [40], or protein changes on the sperm surface after capacitation may allow sperm binding to sephadex particles [41]. Filtration of canine spermatozoa through Sephadex G-15 increased the proportion of viable spermatozoa and decreased the proportion of altered acrosomes compared to the unfiltered sample [37]. Filtered bovine spermatozoa showed improved post-thaw viability compared to unfiltered spermatozoa [42].

There are claims that filtration methods are not only useful for eliminating leukocytes (sources of ROS) and selecting motile spermatozoa, but may also aid selection for morphologically normal [43] and possibly acrosomeintact spermatozoa [39]. In contrast, Januskauskas et al. [42] found no effect on the proportion of spermatozoa with intact acrosomes. The filtrate is not considered to be as clean as for other sperm separation methods [30], presumably because some SP and cellular debris remain in the sample. However, fewer spermatozoa are lost than with other methods, with approximately $63 \%$ recovery rate being reported [42].

5.4. Colloid Centrifugation. In this method, extended semen is centrifuged through a colloid, separating spermatozoa from SP and selecting the spermatozoa that are motile, viable, and have good chromatin integrity. During DGC or SLC, cells move to a point in the gradient that matches their own density-the isopycnic point [44]. By altering the centrifugation force and time, and the physical properties of the colloid, the good quality spermatozoa are allowed to pellet in the bottom of the tube. Until recently, colloid centrifugation was confined solely to DGC, which involved using several layers of colloid of different density (hence "density gradient"), but recently a new method, SLC through a species-specific colloid (Androcoll) was developed by the current authors at the Swedish University for Agricultural Sciences [31], which uses only one layer of Androcoll. Thus, time is saved during preparation and the method can be scaled-up successfully to allow large volumes of ejaculate to be centrifuged [45]. Species-specific formulations of Androcoll (with a suffix to denote the species) have been developed for stallion [31], boar [22], bull [46], dog [47], and cat [48].

Sperm washing is used frequently in the equine breeding industry, either to remove most of the SP prior to extending and cooling the sample, or prior to freezing. However, it should be noted that sperm washing does not improve the quality of the sperm samples since the majority of the spermatozoa are retained during processing. In fact, some of the most motile spermatozoa may be removed with the supernatant [26]. Most of the true selection methods have been tested by researchers at one time or another with the semen of various species but are rarely considered practical for use in the field, mainly because of the large volume of ejaculate or high-sperm concentration involved in preparing sperm doses for animal AI.

In a comparison of SLC and sperm washing for stallion spermatozoa, it was found that sperm washing did not confer any beneficial effect on the sperm samples and, in fact, had a negative effect on sperm motility and chromatin integrity [26]. The SLC-selected samples from the same ejaculates showed both better sperm motility and chromatin integrity, which were maintained over the duration of the experiment (72h).

However, the situation for animal AI is rather different to human ART, since much larger numbers of spermatozoa are required in animal breeding. Thus, with the exception of SLC, the sperm selection methods described above are impractical for incorporating into processing protocols for AI doses in animal breeding. Not only does SLC select good 
quality spermatozoa, as does DGC [31], but also SLC is user friendly and can be scaled up to produce sufficient numbers of spermatozoa even for boar and stallion AI doses [45]. Attempts to scale up DGC have not achieved the same yields as small-scale preparations, although the sperm quality is reported to be good [49]. SLC-selected sperm samples have been shown to be functionally normal in ICSI and AI studies in the horse $[28,50]$, and in bovine IVF [46]. Furthermore, there was an indication that SLC may have advantages over DGC for preparing spermatozoa for ICSI from sperm samples of very poor quality [50].

The major applications for SLC-selection are as follows:

(i) improving sperm quality in AI doses (e.g., stallion [28]);

(ii) increasing the "shelf-life" of in vitro stored sperm samples (stallion [25] and boar [22]);

(iii) removal of pathogens $[51,52]$;

(iv) to improve cryosurvival by removing dead and dying spermatozoa prior to cryopreservation, or used postthawing to select the live spermatozoa $[53,54]$;

(v) to select spermatozoa that are morphologically nor$\mathrm{mal}$ and have good chromatin integrity for AI [55], ICSI [50], or IVF [46], thus increasing the yield of zygotes developing to the blastocyst stage;

(vi) in combination with other methods of sperm sexing, to speed up the process [56].

\section{HBA: Relevance for IVF, ICSI, and AI}

During epididymal maturation, spermatozoa acquire hyaluronic acid binding sites which are necessary for the acquisition of motility and for binding to the ZP/oocyte [57]. Spermatozoa not possessing these binding sites are unable to fertilize oocytes even in IVF. Therefore, detection of the proportion of spermatozoa with these binding sites may indicate the potential fertility of the sperm sample. Since ICSI circumvents all natural barriers to fertilization, the success rate of the technique is dependent on the quality of the spermatozoa selected for injection [58]. Using normal morphology as the sole criterion for selection increases the possibility of using spermatozoa with abnormal chromosomes [59]. Therefore, it was hypothesized that an effective test for selecting normal spermatozoa would increase the success rate of embryo production by ICSI, particularly in pigs [60]. Park et al. [58] reported an increase in ICSI efficiency when boar spermatozoa adhering to droplets of hyaluronic acid (HA) were selected for ICSI, compared to controls using motile spermatozoa that had been slowed down by passage through a solution of polyvinylpyrrolidone (PVP). Although the blastocyst development was the same in the two groups, the frequency of normal diploidy in the group treated with HA was significantly higher $(75.5 \%)$ than in the PVP-group $(68.2 \%)$. The authors concluded that using the HA-binding spermatozoa was superior to morphological sperm selection alone in producing chromosomally normal embryos by
ICSI [58]. Similar results were seen for human ART, where a higher fertilization rate was obtained using HA-selected spermatozoa for ICSI [61]. The latter authors also found a significant inverse correlation between percentage of HA binding and protamine deficiency, DNA fragmentation, and abnormal sperm morphology $(P<.05)$.

Recently an assay was developed for determining the ability of human spermatozoa to bind to hyaluronan. A kit (HBA - the hyaluronan binding assay, Medicult, Denmark) is commercially available for use with human semen samples for IVF, consisting of glass slides coated with hyaluronan. A drop of the sperm sample in phosphate buffered saline is applied to the coated slide and, after a short incubation at ambient temperature $\left(22^{\circ} \mathrm{C}\right)$, the number of spermatozoa bound to the hyaluronan coating is counted. A variant for selecting spermatozoa for ICSI made by the same company consists of hyaluronan-coated dishes (PICSI dishes) from which bound spermatozoa can be selected one by one for use in ICSI.

Although originally developed for human spermatozoa, both the HBA and PICSI dishes have been evaluated for use with stallion spermatozoa destined for AI and ICSI, respectively. When the HBA was used with stallion spermatozoa subjected to cool storage, binding varied between stallions $(11 \%-24 \%$ [62]) but was less than indicated for human spermatozoa (28\%-89\%; [63]). However, the ejaculates used in this trial had been extended in a commercial extender (INRA96; IMV) and stored in the refrigerator overnight, which may have blocked or inactivated some of the binding sites.

Use of the PICSI dishes when selecting spermatozoa for ICSI produced variable results, with lower cleavage rates but a higher proportion of cleaved oocytes developing to the blastocyst stage than for spermatozoa selected by the operator on the basis of apparent "normal" morphology alone [50]. Since previous studies have shown that bovine oocytes can cleave when fertilized with chromosomally abnormal bull spermatozoa, but development is subsequently blocked [64], it would appear that the spermatozoa binding in the PICSI dishes did have a better chance of being chromosomally normal than those selected on the basis of subjective morphology assessment by a skilled operator.

\section{Sex Selection}

For many centuries, animal breeders and researchers have endeavoured to control the sex of the offspring born, for various reasons. Initially male offspring were preferred, for example, for beef production, because of the better feed conversion efficiency and lean-to-fat ratio of males whereas females were preferred for dairy purposes, except that some males of high-genetic merit were still required as sires. Current husbandry practices in the pig industry favour samesex groups on welfare grounds (to prevent fighting) and because of a more even live-weight gain, with all members of the group reaching slaughter weight within a specified time. However, if male pigs are left entire, the meat develops "boar taint" as the animal reaches sexual maturity, which is 
considered undesirable by the consumer. Therefore, young males must be castrated, which has welfare considerations. One mechanism for avoiding castration would be to produce only female piglets in litters destined for intensive meat production, obtained by using sexed sperm doses for AI.

Many methods have been proposed for separating Xand Y-chromosome bearing spermatozoa, based on physical properties, for example, size of the sperm head, or functional properties, for example, swimming speed. However, the only method which has been shown to work reliably is that of selection and separation of spermatozoa whose DNA is stained vitally with a bis-benzimidazole dye, H33342 [65], and separated into X- and Y-chromosome bearing groups using the sorting capacity of a flow cytometer [66, 67]. This method functions because the $\mathrm{X}$ chromosome is larger than the $Y$ and therefore takes up more of the DNA-specific stain. In cattle, for example, the difference in DNA content between the $\mathrm{X}$ and $\mathrm{Y}$ chromosome is approximately $4.2 \%$, whereas for pigs it is approximately $3.8 \%$. However, the process of sorting sufficient numbers for an insemination dose in the flow cytometer is long since the stained spermatozoa must pass one at a time through a laser beam for detection of their DNA content. Although a commercial enterprise exists for sexing bull spermatozoa, the method is considered too expensive for commercial application in pig breeding, where much larger numbers of spermatozoa are needed per AI dose than for cattle. Therefore, flow cytometric sperm sexing for AI has been confined largely to the cattle breeding industry, and for ICSI in the equine breeding industry.

Even in the bovine breeding industry, flow cytometric sperm sexing has not fulfilled its original promise: sexed sperm doses are too expensive for widespread application, the fertility of sexed sperm doses is lower than unsexed sperm doses [68], which is not merely a reflection of the lower sperm numbers used but may be attributed to impaired sperm function caused by the sexing process [69]. It is worth noting that the first method $[65,66]$ developed for sperm sexing used a lower concentration of the vital dye, Hoechst 33342 than the method described later [67]. The higher dye concentration was linked to chromosomal abnormalities in rabbits, particularly in conjunction with laser illumination [70] whereas the lower concentration was used to produce 5 successive generations of rabbits without any evidence of fertility problems [71]. Since food dye is added to sperm samples stained with the higher concentration of dye to quench the excessive fluorescence [72], it would seem to be better to use lower concentrations of Hoechst 33342 initially to reduce the possibility of negative effects.

Furthermore, the flow cytometric technique is not applicable to all bulls; there appears to be a difference in "sortability" between individuals [69]. A similar variation between males was observed for stallions [73]; the latter authors attributed the variation in "sortability" to differences in the number of dead spermatozoa present in the sample. Therefore, there is still a need in the animal breeding industry to develop a technique for sperm sexing that would provide sufficient spermatozoa for AI doses, does not compromise sperm fertility, and is widely applicable to a range of individuals and species.
One possible alternative to flow cytometric separation is colloid centrifugation, either density gradient centrifugation (DGC) or the recently developed technique of single layer centrifugation (SLC). Since the X chromosome is larger than the $\mathrm{Y}$, while no difference has been detected in the volume of the sperm head, there is a theoretical difference in density between the two sperm types. Thus, if there is no other factor contributing to sperm density, it should be possible to separate the two types using colloid centrifugation. However, spermatozoa change in density as they mature due to changes in the composition of their membranes, and the distribution in density due to the maturity of the spermatozoa overlaps that of the sex chromosome. Therefore, it is not certain that it would be possible to sex spermatozoa in sufficient numbers with colloid centrifugation to be useful for AI doses. To date, attempts have been made to sex small numbers of bull spermatozoa with DGC for subsequent IVF [74, 75]. The authors of the first paper claimed a shift in the sex ratio of embryos produced whereas the authors of the second found no change in the sex ratio.

Another possibility for sperm sexing is that sperm surface proteins may be sex linked [76]. Recent research has identified proteins on either X- or Y-chromosome bearing spermatozoa. The use of antibodies to these sperm surface proteins may enable the two subpopulations of spermatozoa to be separated.

A combination of techniques would also be relevant for sperm sexing. Thus, the speed of flow sorting can be increased by first removing the dead and dying spermatozoa from the population, for example by DGC or SLC [77]. Such a combination may increase the "sortability" of sperm samples. Since SCL with Androcoll appears to be better at removing spermatozoa with damaged chromatin, at least from stallion ejaculates [50], and is a simpler technique to use than DGC, it would appear to be advantageous to combine SLC with Androcoll and flow cytometry for producing sexed sperm doses for AI.

\section{Conclusion}

There are many mechanisms by which spermatozoa may be "selected" for use in ART, varying from a relatively crude fractionation of boar semen as it is being ejaculated to a highly refined process based on the possession/expression of certain cell surface molecules. Any selection mechanisms taking place in vitro must not harm the spermatozoa or modulate their interaction with the female reproductive tract if fertility is to be maintained. Biomimetic selection of the best quality spermatozoa for AI or for cryopreservation could improve pregnancy rates and may help to reverse the decline in fertility seen in several domestic species over the recent decades, for example in dairy cattle [78] and horses [79]. Similarly, selection for hyaluronic acid binding sites may enable the most mature spermatozoa to be selected for IVF or ICSI, resulting in a more efficient production of blastocysts than at present, or even help to identify the best males to be selected as breeding sires. Finally, as previously discussed in this paper, the animal breeding industry is still waiting 
for an effective, practical method of sexing spermatozoa, which is applicable to the semen of all males. Perhaps this achievement will become the most notable contribution to the animal breeding industry of the 21st century, as AI and sperm cryopreservation were to the 20th century.

\section{Acknowledgment}

J. Morrell is funded by the Swedish Farmers' Foundation, Stockholm (V0750239), and the Swedish Ministry of Agriculture, Jönköping (31-3389/09).

\section{References}

[1] S. S. Suarez, "Interactions of spermatozoa with the female reproductive tract: inspiration for assisted reproduction," Reproduction, Fertility and Development, vol. 19, no. 1, pp. 103-110, 2007.

[2] D. Rath, H. J. Schuberth, P. Coy, and U. Taylor, "Sperm interactions from insemination to fertilization," Reproduction in Domestic Animals, vol. 43, no. 5, pp. 2-11, 2008.

[3] H. Rodriguez-Martinez, J. Hultgren, R. Båge, et al., "Reproductive performance in high-producing dairy cows: can we sustain it under current practice?" in IVIS Reviews in Veterinary Medicine, I.V.I.S, Ed., International Veterinary Information Service, Ithaca, NY, USA, 2008, http://www.ivis.org/.

[4] C. Barros, P. Vigil, E. Herrera, B. Arguello, and R. Walker, "Selection of morphologically abnormal sperm by human cervical mucus," Archives of Andrology, vol. 12, pp. 95-107, 1984.

[5] K. J. Mullins and R. G. Saacke, "Study of the functional anatomy of bovine cervical mucosa with special reference to mucus secretion and sperm transport," Anatomical Record, vol. 225, no. 2, pp. 106-117, 1989.

[6] H. Rodriguez-Martinez, L. Nicander, S. Viring, S. Einarsson, and K. Larsson, "Ultrastructure of the uterotubal junction in preovulatory pigs," Anatomia, Histologia, Embryologia, vol. 19, no. 1, pp. 16-36, 1990.

[7] U. Taylor, D. Rath, H. Zerbe, and H.-J. Schuberth, "Interaction of intact porcine spermatozoa with epithelial cells and neutrophilic granulocytes during uterine passage," Reproduction in Domestic Animals, vol. 43, no. 2, pp. 166-175, 2008.

[8] U. Taylor, H. J. Schuberth, D. Rath, H. W. Michelmann, C. Sauter-Louis, and H. Zerbe, "Influence of inseminate components on porcine leucocyte migration in vitro and in vivo after pre- and post-ovulatory insemination," Reproduction in Domestic Animals, vol. 44, no. 2, pp. 180-188, 2009.

[9] J. N. Mburu, H. Rodriguez-Martinez, and S. Einarsson, "Changes in sperm ultrastructure and localisation in the porcine oviduct around ovulation," Animal Reproduction Science, vol. 47, no. 1-2, pp. 137-148, 1997.

[10] J. N. Mburu, S. Einarsson, N. Lundeheim, and H. RodriguezMartinez, "Distribution, number and membrane integrity of spermatozoa in the pig oviduct in relation to spontaneous ovulation," Animal Reproduction Science, vol. 45, no. 1-2, pp. 109-121, 1996.

[11] E. Töpfer-Petersen, A. Wagner, J. Friedrich et al., "Function of the mammalian oviductal sperm reservoir," Journal of Experimental Zoology, vol. 292, no. 2, pp. 210-215, 2002.

[12] F. Ardón, D. Helms, E. Sahin, H. Bollwein, E. Töpfer-Petersen, and D. Waberski, "Chromatin-unstable boar spermatozoa have little chance of reaching oocytes in vivo," Reproduction, vol. 135, no. 4, pp. 461-470, 2008.

[13] M. J. Munuce, A. Serravalle, A. M. Caille et al., "Human tubal secretion can modify the affinity of human spermatozoa for the zona pellucida," Fertility and Sterility, vol. 91, no. 2, pp. 407-413, 2009.

[14] P. Talbot, B. D. Shur, and D. G. Myles, "Cell adhesion and fertilization: steps in oocyte transport, sperm-zona pellucida interactions, and sperm-egg fusion," Biology of Reproduction, vol. 68 , no. 1, pp. 1-9, 2003.

[15] W. M. Maxwell, S. P. de Graaf, R. H. Ghaoui, and G. Evans, "Seminal plasma effects on sperm handling and female fertility," Society of Reproduction and Fertility, vol. 64, pp. 1338, 2007.

[16] I. Caballero, J. M. Vazquez, E. M. García et al., "Major proteins of boar seminal plasma as a tool for biotechnological preservation of spermatozoa," Theriogenology, vol. 70, no. 8, pp. 1352-1355, 2008.

[17] J. M. Vazquez, I. Parrilla, M. A. Gil et al., "Improving the efficiency of insemination with sex-sorted spermatozoa," Reproduction in Domestic Animals, vol. 43, no. 4, pp. 1-8, 2008.

[18] P. C. Rodriguez, L. B. Valdez, T. Zaobornyj, A. Boveris, and M. T. Beconi, "Nitric oxide and superoxide anion production during heparin-induced capacitation in cryopreserved bovine spermatozoa," Reproduction in Domestic Animals. In press.

[19] U. Lavon and J. C. Boursnell, "The split ejaculate of the boar: contributions of the epididymides and seminal vesicles," Journal of Reproduction and Fertility, vol. 42, no. 3, pp. 541552, 1975.

[20] F. Saravia, M. Wallgren, and H. Rodríguez-Martínez, "Freezing of boar semen can be simplified by handling a specific portion of the ejaculate with a shorter procedure and MiniFlatPack packaging," Animal Reproduction Science, vol. 117, no. 3-4, pp. 279-287, 2010.

[21] F. Saravia, M. Wallgren, A. Johannisson et al., "Exposure to the seminal plasma of different portions of the boar ejaculate modulates the survival of spermatozoa cryopreserved in MiniFlatPacks," Theriogenology, vol. 71, no. 4, pp. 662-675, 2009.

[22] J. M. Morrell, F. Saravia, M. van Wienen, H. RodriguezMartinez, and M. Wallgren, "Sperm survival following colloid centrifugation varies according to the part of the sperm-rich fraction used," Society of Reproduction and Fertility, vol. 66, pp. 85-86, 2009.

[23] M. Kareskoski and T. Katila, "Components of stallion seminal plasma and the effects of seminal plasma on sperm longevity," Animal Reproduction Science, vol. 107, no. 3-4, pp. 249-256, 2008.

[24] C. Aurich, "Factors affecting the plasma membrane function of cooled-stored stallion spermatozoa," Animal Reproduction Science, vol. 89, no. 1-4, pp. 65-75, 2005.

[25] J. M. Morrell, A.-M. Dalin, and H. Rodriguez-Martinez, "Comparison of density gradient and single layer centrifugation of stallion spermatozoa: yield, motility and survival," Equine Veterinary Journal, vol. 41, no. 1, pp. 53-58, 2009.

[26] J. M. Morrell, H. Rodriguez-Martinez, and A. Johannisson, "Single layer centrifugation of stallion spermatozoa improves sperm quality compared with sperm washing," Reproductive Biomedicine. In press.

[27] M. H. T. Troedsson, A. Desvousges, A. S. Alghamdi et al., "Components in seminal plasma regulating sperm transport and elimination," Animal Reproduction Science, vol. 89, no. 14, pp. 171-186, 2005. 
[28] G. Mari, E. Iacono, G. Kutvölgyi, B. Mislei, H. RodriguezMartinez, and J. M. Morrell, "Stallion spermatozoa prepared by Single Layer Centrifugation with Androcoll-E are capable of fertilisation in vivo," Reproduction in Domestic Animals. In press.

[29] J. M. Morrell and H. Rodriguez-Martinez, "Biomimetic techniques for improving sperm quality in animal breeding: a review," Open Andrology Journal, vol. 1, pp. 1-9, 2009.

[30] R. R. Henkel and W.-B. Schill, "Sperm preparation for ART," Reproductive Biology and Endocrinology, vol. 1, article 108, 2003.

[31] J. M. Morrell, A.-M. Dalin, and H. Rodriguez-Martinez, "Prolongation of stallion sperm survival by centrifugation through coated silica colloids: a preliminary study," Animal Reproduction, vol. 5, pp. 121-126, 2008.

[32] L. Björndahl, M. Mohammadieh, M. Pourian, I. Söderlund, and U. Kvist, "Contamination by seminal plasma andrology lab corner factors during sperm selection," Journal of Andrology, vol. 26, no. 2, pp. 170-173, 2005.

[33] T. Hallap, M. Håård, U. Jaakma, B. Larsson, and H. RodriguezMartinez, "Does cleansing of frozen-thawed bull semen before assessment provide samples that relate better to potential fertility?” Theriogenology, vol. 62, no. 3-4, pp. 702-713, 2004.

[34] D. Mortimer, "Sperm preparation methods," Journal of Andrology, vol. 21, no. 3, pp. 357-366, 2000.

[35] T. Somfai, S. Bodó, S. Nagy et al., "Effect of swim up and Percoll treatment on viability and acrosome integrity of frozen-thawed bull spermatozoa," Reproduction in Domestic Animals, vol. 37, no. 5, pp. 285-290, 2002.

[36] M. Shamsuddin and H. Rodriguez-Martinez, "A simple, nontraumatic swim-up method for the selection of spermatozoa for in vitro fertilization in the bovine," Animal Reproduction Science, vol. 36, no. 1-2, pp. 61-75, 1994.

[37] T. Mogas, T. Rigau, J. Piedrafita, S. Bonet, and J. E. RodriguezGil, "Effect of column filtration upon the quality parameters of fresh dog semen," Theriogenology, vol. 50, no. 8, pp. 1171$1189,1998$.

[38] E. Bussalleu, E. Pinart, M. M. Rivera et al., "Effects of filtration of semen doses from subfertile boars through neuter Sephadex columns," Reproduction in Domestic Animals, vol. 43, no. 1, pp. 48-52, 2008.

[39] M. Anzar, E. F. Graham, and N. Iqbal, "Post-thaw plasma membrane integrity of bull spermatozoa separated with a sephadex ion-exchange column," Theriogenology, vol. 47, no. 4, pp. 845-856, 1997.

[40] Z. Ahmad, M. Anzar, M. Shahab, N. Ahmad, and S. M. H. Andrabi, "Sephadex and sephadex ion-exchange filtration improves the quality and freezability of low-grade buffalo semen ejaculates," Theriogenology, vol. 59, no. 5-6, pp. 1189 1202, 2003.

[41] J. C. Samper, D. W. Hamilton, J. L. Pryor, K. J. Loseth, M. H. T. Troedsson, and B. G. Crabo, "Mechanism of sephadex trapping of capacitated stallion spermatozoa," Biology of Reproduction, pp. 729-737, 1995.

[42] A. Januskauskas, K. Lukoseviciute, S. Nagy, A. Johannisson, and H. Rodriguez-Martinez, "Assessment of the efficacy of Sephadex G-15 filtration of bovine spermatozoa for cryopreservation," Theriogenology, vol. 63, no. 1, pp. 160-178, 2005.

[43] N. M. Ibrahim, D. N. Foster, and B. G. Crabo, "Localization of clusterin on freeze-preserved bull spermatozoa before and after glass wool-sephadex filtration," Journal of Andrology, vol. 22, no. 5, pp. 891-902, 2001.
[44] H. Pertoft, "Fractionation of cells and subcellular particles with Percoll," Journal of Biochemical and Biophysical Methods, vol. 44, no. 1-2, pp. 1-30, 2000.

[45] J. M. Morrell, A. Johannisson, A.-M. Dalin, and H. RodriguezMartinez, "Single-layer centrifugation with Androcoll ${ }^{T M}-\mathrm{E}$ can be scaled up to allow large volumes of stallion ejaculate to be processed easily," Theriogenology, vol. 72, no. 6, pp. 879884, 2009.

[46] M. Thys, L. Vandaele, J. Morrell et al., "In vitro fertilizing capacity of frozen-thawed bull spermatozoa selected by singlelayer (Glycidoxypropyltrimethoxysilane) silane-coated silica colloidal centrifugation," Reproduction in Domestic Animals, vol. 44, no. 3, pp. 390-394, 2009.

[47] J. M. Morrell, H. Rodriguez-Martinez, and C. Linde Forsberg, "Single layer centrifugation on a colloid selects motile and morphologically normal spermatozoa from dog semen: preliminary results," Reproduction in Domestic Animals, vol. 43, article 61, 2008, abstract P29.

[48] K. Chatdarong, P. Thuwanut, and J. M. Morrell, "Singlelayer centrifugation through colloid selects improved quality of epididymal cat sperm," Theriogenology, vol. 73, no. 9, pp. 1284-1292, 2010.

[49] A. J. Edmond, A. R. Teague, S. P. Brinsko, et al., "Effect of density gradient centrifugation on quality and recovery of equine spermatozoa," Animal Reproduction Science, vol. 107, article 318, 2008, abstract 16.

[50] J. M. Morrell, S. Colleoni, I. Lagutina, H. Rodriguez-Martinez, G. Lazzari, and C. Galli, "Stallion spermatozoa selected by Single Layer Centrifugation with Androcoll ${ }^{T M}$-E have normal functionality after ICSI," Animal Reproduction Science, vol. 121S, pp. S196-S197, 2010.

[51] M. Wallgren, H. Rodriguez-Martinez, and J. M. Morrell, "Single Layer Centrifugation with Androcoll ${ }^{T M}$-P removes, or substantially reduces, bacterial contamination in boar sperm samples," in Proceedings of the 11th International Symposium on Spermatology, Okinawa, Japan, June 2010.

[52] J. M. Morrell, "Prevention of pathogen transmission through breeding in horses," Reproduction in Domestic Animals, vol. 44, supplement 3, 2009, abstract 15.3.

[53] B. Macías García, J. M. Morrell, C. Ortega-Ferrusola et al., "Centrifugation on a single layer of colloid selects improved quality spermatozoa from frozen-thawed stallion semen," Animal Reproduction Science, vol. 114, no. 1-3, pp. 193-202, 2009.

[54] B. M. García, L. G. Fernández, J. Morrell et al., "Singlelayer centrifugation through colloid positively modifies the sperm subpopulation structure of frozen-thawed stallion spermatozoa," Reproduction in Domestic Animals, vol. 44, no. 3, pp. 523-526, 2009.

[55] J. Morrell, A. Johannisson, A.-M. Dalin, and H. RodriguezMartinez, "Morphology and chromatin integrity of stallion spermatozoa prepared by density gradient and single layer centrifugation through silica colloids," Reproduction in Domestic Animals, vol. 44, no. 3, pp. 512-517, 2009.

[56] W. M. C. Maxwell, I. Parrilla, I. Caballero et al., "Retained functional integrity of bull spermatozoa after double freezing and thawing using PureSperm $(\mathrm{B}$ ) density gradient centrifugation," Reproduction in Domestic Animals, vol. 42, no. 5, pp. 489-494, 2007.

[57] G. Huszar, C. C. Ozenci, S. Cayli, Z. Zavaczki, E. Hansch, and L. Vigue, "Hyaluronic acid binding by human sperm indicates cellular maturity, viability, and unreacted acrosomal status," Fertility and Sterility, vol. 79, no. 3, pp. 1616-1624, 2003. 
[58] C. Y. Park, S. J. Uhm, S. J. Song, et al., "Increase of ICSI efficiency with hyaluronic acid binding sperm for low aneuploidy frequency in pig," Theriogenology, vol. 64, no. 5, pp. 1158-1169, 2005.

[59] W. Rohini Edirisinghe, A. Murch, S. Junk, and J. L. Yovich, "Cytogenetic abnormalities of unfertilized oocytes generated from in-vitro fertilization and intracytoplasmic sperm injection: a double-blind study," Human Reproduction, vol. 12, no. 12, pp. 2784-2791, 1997.

[60] M. Nakai, N. Kashiwazaski, A. Takizawa et al., "Viable piglets generated from porcine oocytes matured in vitro and fertilized by intracytoplasmic sperm head injection," Biology of Reproduction, vol. 68, no. 3, pp. 1003-1008, 2003.

[61] M. H. Nasr-Esfahani, S. Razavi, A. A. Vahdati, F. Fathi, and M. Tavalaee, "Evaluation of sperm selection procedure based on hyaluronic acid binding ability on ICSI outcome," Journal of Assisted Reproduction and Genetics, vol. 25, no. 5, pp. 197-203, 2008.

[62] J. M. Morrell, S. Meurling, and H. Rodriguez-Martinez, "Can the HBA be used predictively for stallion spermatozoa," in Proceedings of the 11th International Symposium on Spermatology, Okinawa, Japan, June 2010.

[63] H. Ye, G.-N. Huang, Y. Gao, and D. Y. Liu, "Relationship between human sperm-hyaluronan binding assay and fertilization rate in conventional in vitro fertilization," Human Reproduction, vol. 21, no. 6, pp. 1545-1550, 2006.

[64] A. N. Fatehi, M. M. Bevers, E. Schoevers, B. A. J. Roelen, B. Colenbrander, and B. M. Gadella, "DNA damage in bovine sperm does not block fertilization and early embryonic development but induces apoptosis after the first cleavages," Journal of Andrology, vol. 27, no. 2, pp. 176-188, 2006.

[65] K. D. Keeler, N. M. Mackenzie, and D. W. Dresser, "Flow microfluorometric analysis of living spermatozoa stained with Hoechst 33342," Journal of Reproduction and Fertility, vol. 68, no. 1, pp. 205-212, 1983.

[66] J. M. Morrell, K. D. Keeler, D. E. Noakes, N. M. Mackenzie, and D. W. Dresser, "Sexing of sperm by flow cytometry," Veterinary Record, vol. 122, no. 14, pp. 322-324, 1988.

[67] L. A. Johnson, J. P. Flook, and H. W. Hawk, "Sex preselection in rabbits: live births from $\mathrm{X}$ and $\mathrm{Y}$ sperm separated by DNA and cell sorting," Biology of Reproduction, vol. 41, no. 2, pp. 199-203, 1989.

[68] G. E. Seidel Jr., "Overview of sexing sperm," Theriogenology, vol. 68, no. 3, pp. 443-446, 2007.

[69] S. L. Underwood, R. Bathgate, M. Ebsworth, W. M. C. Maxwell, and G. Evans, "Pregnancy loss in heifers after artificial insemination with frozen-thawed, sex-sorted, refrozen-thawed dairy bull sperm," Animal Reproduction Science, vol. 118, no. 1, pp. 7-12, 2010.

[70] B. L. Libbus, S. D. Perreault, L. A. Johnson, and D. Pinkel, "Incidence of chromosome aberrations in mammalian sperm stained with Hoechst 33342 and UV-laser irradiated during flow sorting," Mutation Research, vol. 182, no. 5, pp. 265-274, 1987.

[71] J. M. Morrell and D. W. Dresser, "Offspring from inseminations with mammalian sperm stained with Hoechst 33342, either with or without flow cytometry," Mutation Research, vol. 224, no. 2, pp. 177-183, 1989.

[72] L. A. Johnson and G. R. Welch, "Sex preselection: high-speed flow cytometric sorting of $\mathrm{X}$ and $\mathrm{Y}$ sperm for maximum efficiency," Theriogenology, vol. 52, no. 8, pp. 1323-1341, 1999.
[73] J. R. Clulow, G. Evans, L. H. A. Morris, and W. M. C. Maxwell, "Factors influencing the "sortability" of stallion spermatozoa into X- and Y-chromosome bearing populations," Animal Reproduction Science, vol. 113, no. 1-4, pp. 220-228, 2009.

[74] C. A. Wolf, K. E. Brass, M. I. B. Rubin, S. E. Pozzobon, F. D. Mozzaquatro, and F. D. de la Corte, "The effect of sperm selection by Percoll or swim up on the sex ratio of in vitro produced bovine embryos," Animal Reproduction, vol. 5, pp. 110-115, 2008.

[75] G. M. Machado, J. O. Carvalho, E. S. Filho et al., "Effect of Percoll volume, duration and force of centrifugation, on in vitro production and sex ratio of bovine embryos," Theriogenology, vol. 71, no. 8, pp. 1289-1297, 2009.

[76] S. R. Blecher, R. Howie, S. Li, J. Detmar, and L. M. Blahut, "A new approach to immunological sexing of sperm," Theriogenology, vol. 52, no. 8, pp. 1309-1321, 1999.

[77] F. K. Hollinshead, J. K. O'Brien, W. M. C. Maxwell, and G. Evans, "Assessment of in vitro sperm characteristics after flow cytometric sorting of frozen-thawed bull spermatozoa," Theriogenology, vol. 62, no. 5, pp. 958-968, 2004.

[78] H. Dobson, R. F. Smith, M. D. Royal, C. H. Knight, and I. M. Sheldon, "The high-producing dairy cow and its reproductive performance," Reproduction in Domestic Animals, vol. 42, no. 2, pp. 17-23, 2007.

[79] T. Katila, T. Reilas, K. Nivola, T. Peltonen, and A.-M. Virtala, "A 15-year survey of reproductive efficiency of Standardbred and Finnhorse trotters in Finland-descriptive results," Acta Veterinaria Scandinavica. In press. 

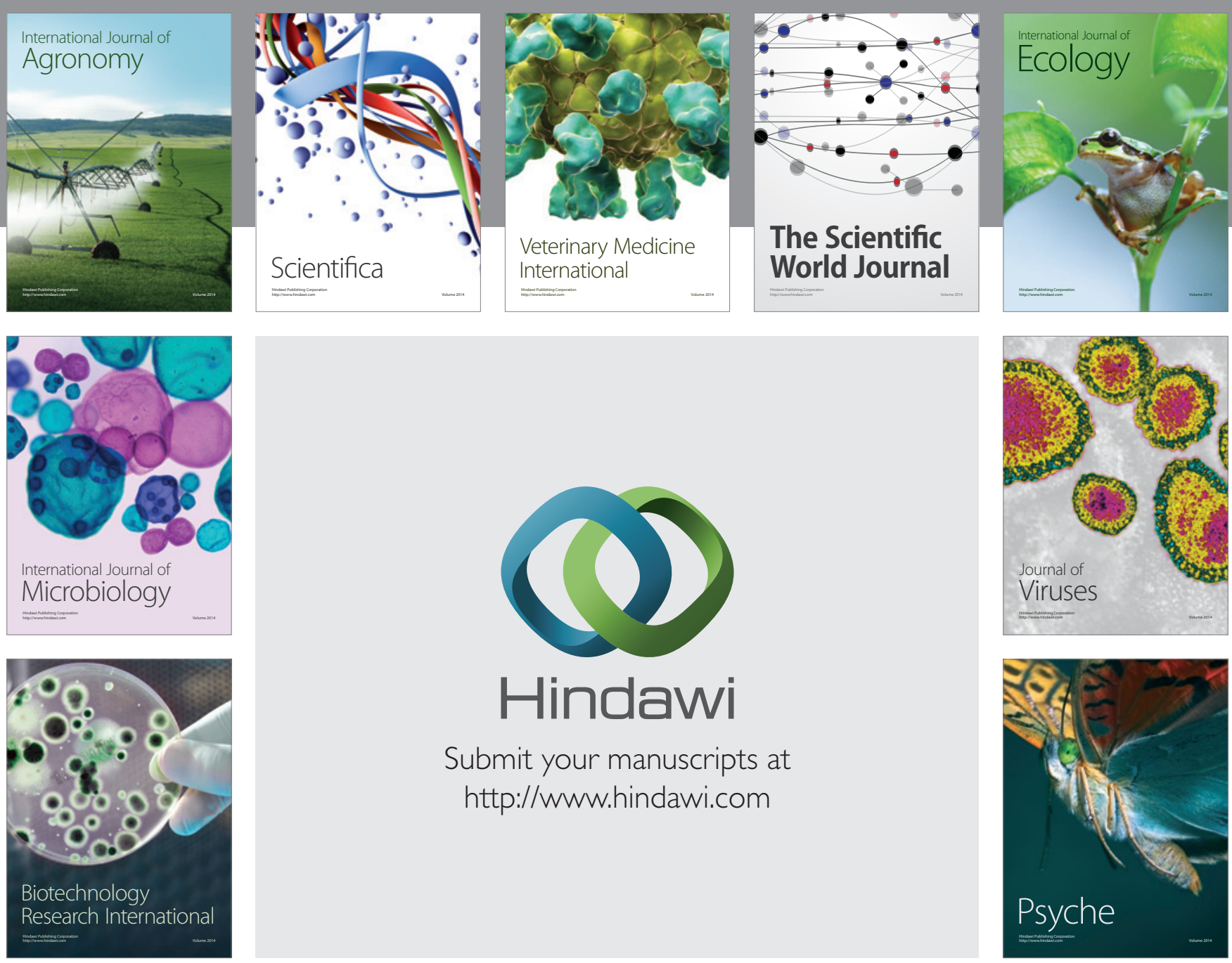

Submit your manuscripts at

http://www.hindawi.com
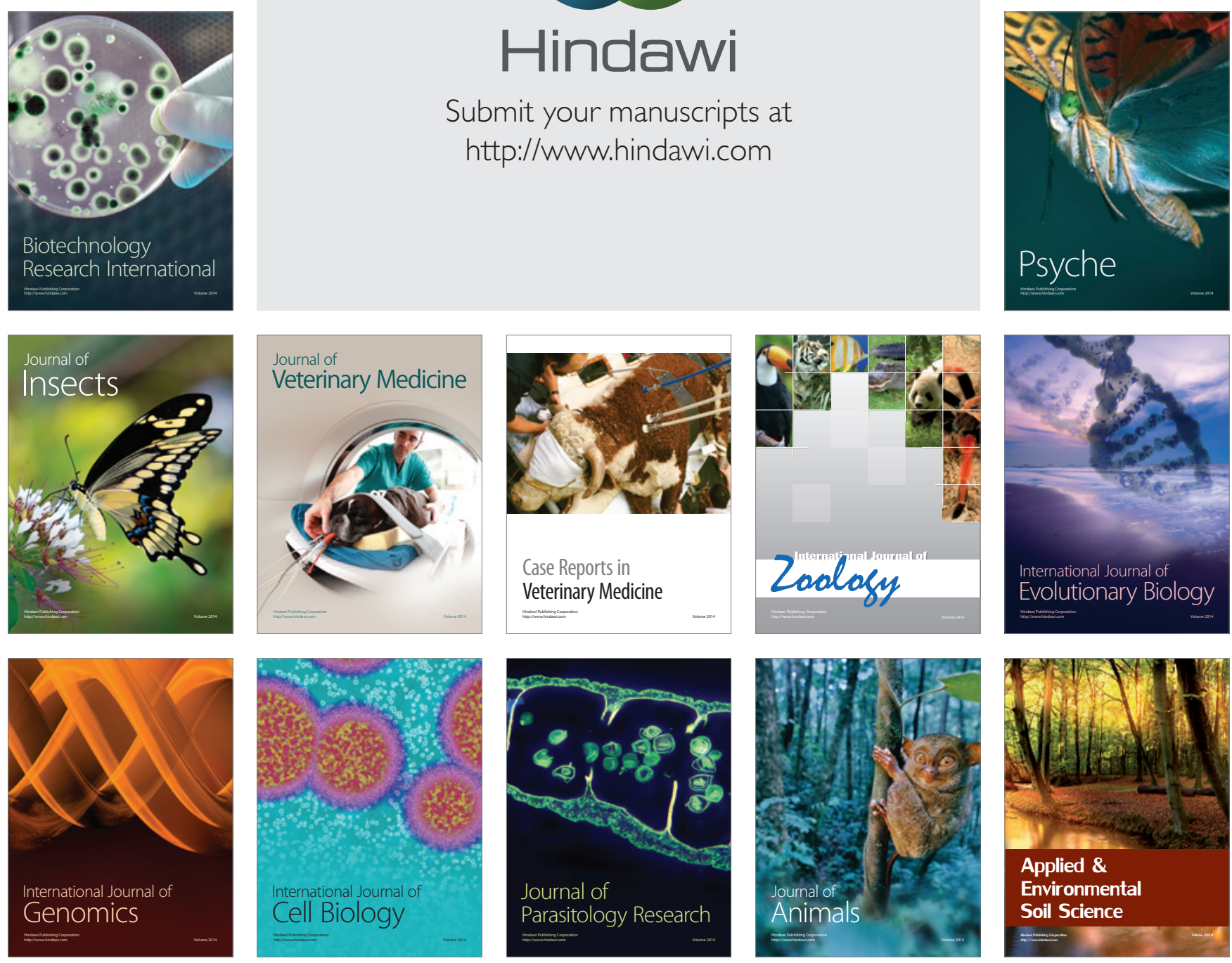